Research Paper

\title{
Asiatic Acid Protects against Cardiac Hypertrophy through Activating AMPKa Signalling Pathway
}

\author{
Zhen-Guo Ma1,2, Jia Dai ${ }^{1,2}$, Wen-Ying Wei ${ }^{1,2}$, Wen-Bin Zhang ${ }^{1,2}$, Si-Chi Xu ${ }^{1,2}$, Hai-Han Liao ${ }^{1,2}$, \\ Zheng Yang1,2, Qi-Zhu Tang ${ }^{1,2}$ \\ 1. Department of Cardiology, Renmin Hospital of Wuhan University, Wuhan, China; \\ 2. Cardiovascular Research Institute of Wuhan University, Wuhan, China. \\ $\triangle$ Corresponding author: Qi-Zhu Tang, Department of Cardiology, Renmin Hospital of Wuhan University; Cardiovascular Research Institute, Wuhan \\ University at Jiefang Road 238, Wuhan 430060, China. Tel.: +86 27 88073385; Fax: +86 27 88042292. E-mail: qztang@whu.edu.cn. \\ (1) Ivyspring International Publisher. Reproduction is permitted for personal, noncommercial use, provided that the article is in whole, unmodified, and properly cited. See \\ http://ivyspring.com/terms for terms and conditions.
}

Received: 2015.10.24; Accepted: 2016.03.23; Published: 2016.05.25

\begin{abstract}
Background: AMP-activated protein kinase $\alpha(A M P K \alpha)$ is closely involved in the process of cardiac hypertrophy. Asiatic acid (AA), a pentacyclic triterpene, was found to activate AMPK $\alpha$ in our preliminary experiment. However, its effects on the development of cardiac hypertrophy remain unclear. The present study was to determine whether AA could protect against cardiac hypertrophy.

Methods: Mice subjected to aortic banding were orally given AA (10 or $30 \mathrm{mg} / \mathrm{kg}$ ) for 7 weeks. In the inhibitory experiment, Compound $C$ was intraperitoneally injected for 3 weeks after surgery.

Results: Our results showed that AA markedly inhibited hypertrophic responses induced by pressure overload or angiotensin II. AA also suppressed cardiac fibrosis in vivo and accumulation of collagen in vitro. The protective effects of AA were mediated by activation of AMPKa and inhibition of the mammalian target of rapamycin (mTOR) pathway and extracellular signal-regulated kinase (ERK) in vivo and in vitro. However, AA lost the protective effects after AMPKa inhibition or gene deficiency.

Conclusions: AA protects against cardiac hypertrophy by activating AMPKa, and has the potential to be used for the treatment of heart failure.
\end{abstract}

Key words: AMPKa; Asiatic acid; Cardiac fibrosis; Cardiac hypertrophy.

\section{Introduction}

Cardiac hypertrophy is characterized by an increase in size of cardiac myocyte and protein synthesis, abnormal expression of fetal gene, and excessive deposition of extracellular matrix [1-2]. Cardiac hypertrophy, which can result in heart failure and ventricular arrhythmias, is a risk factor for cardiovascular events and death [3]. The precise mechanisms controlling the process of cardiac hypertrophy are incompletely understood, but emerging data implicate the key roles for the mammalian target of rapamycin (mTOR) and extracellular signal-regulated kinase (ERK) [4-5].

Activated mTOR promotes the activation of ribosomal protein S6 kinase (P70S6K), directly regulating protein synthesis and contributing to the process of cardiac hypertrophy [6]. Conversely, inhibition of mTOR signaling with rapamycin attenuates established cardiac hypertrophy [4]. ERK regulates development of hypertrophy through the induction of the expression of hypertrophy-related genes [5]. It is reported that MTOR and ERK can be regulated by AMP-activated protein kinase a (AMPKa), which is an energy sensor of heart [6-7]. AMPKa is also closely involved in the process of cardiac hypertrophy. Activation of AMPKa by pharmacological compounds have been shown to inhibit cardiac hypertrophy [8-9]. Therefore, drugs that selectively activate AMPKa and inhibit mTOR 
and ERK could be of great therapeutic interest for the treatment of cardiac hypertrophy.

Asiatic acid (AA), a chemically characterized pentacyclic triterpene, naturally exists in many edible vegetables and fruits including brown mustard, basil, daylily, hawthorn fruit and spinach [10-11]. Previous study found that dietary intake of AA increased their bioavailability in the heart [10]. Accumulating evidence indicated that AA possessed a number of pharmacological activities. AA has been reported to reduce islet fibrosis, suppress mitochondria-mediated inflammasome activation, ameliorate hepatic lipid accumulation and insulin resistance, and attenuate infarct volume after focal cerebral ischemia [12-15]. AA also alleviated cardiovascular remodeling in hypertensive rat and protected against diabetic cardiomyopathy [16-17]. In our preliminary experiment, we found AA activated AMPKa in the heart. However, the effects of AA on cardiac hypertrophy and the precise mechanisms still remain unclear. Therefore, this study was to determine whether AA could alleviate hypertrophy of neonatal rat cardiac myocytes and cardiac hypertrophy induced by long-time pressure overload, as well as to reveal the precise mechanisms underlying the putative effects.

\section{Materials and methods}

\subsection{Materials}

AA was purchased from Shanghai Winherb Medical Co. (Shanghai, China), who specified above 98.5\% purity confirmed by HPLC. Angiotensin II (Ang II, A9525) and Compound C (CpC, P5499) were purchased from Sigma-Aldrich. Anti-GAPDH (sc-25778) was obtained from Santa Cruz Biotechnology. Anti-AMPKa (\#2603P), anti-phospho-AMPKa (\#2535), anti-phospho-mTOR (\#2971), anti-mTOR (\#2983), anti-P70S6K (\#2708), anti-phospho-P70S6K (\#9234P), anti-ribosomal protein S6 (S6, \#2217), anti-phospho-S6 (\#5364P), anti-acetyl-CoA carboxylase (ACC, \#3676), anti-phospho-ACC (\#3661), anti-ERK (\#4695), and anti-phospho-ERK(\#4370) were obtained from Cell Signalling Technology. Anti-a-actin was purchased from Millipore. The secondary antibody was purchased from LI-COR Biosciences (at 1:10,000 dilution). All other chemicals were of analytical grade.

\subsection{Animals and treatments}

All animal experimental procedures were approved by the Guidelines for the Care and Use of Laboratory Animals of the Chinese Animal Welfare Committee and the guidelines of our hospital. All surgeries and subsequent analyses were performed in a blind fashion for all groups. C57/B6 mice (8-10 week-old; male body weight: $25.5 \pm 2 \mathrm{~g}$ ) were subjected to aortic banding (AB) according to the previous article [18]. The source of AMPKa2 knockout mice was described in our previous article [19]. Doppler analysis was performed to ensure the appropriate constriction of the aorta had been induced. AA was dissolved in $0.1 \%$ DMSO for in vitro experiments and in $1 \% \mathrm{CMCNa}$ for in vivo experiments. One week after being subjected to $\mathrm{AB}$, mice were given $\mathrm{AA}$ (10 $\mathrm{mg} / \mathrm{kg}$ or $30 \mathrm{mg} / \mathrm{kg}$ ) for 7 weeks, and animals in the control group were given equal volumes of vehicle. To inhibit AMPKa, CpC (20mg/ kg, every other day) was injected for 3 weeks beginning from 1 week after surgery to 4 weeks after surgery. In the inhibition experiment, the $A B$ surgery lasts for 4 weeks. Mice were subjected to AA $(30 \mathrm{mg} / \mathrm{kg})$ for 3 weeks from 1 week after surgery to 4 weeks after surgery. AMPKa2 knockout mice were subjected to AA $(30 \mathrm{mg} / \mathrm{kg})$ treatment for 3 weeks from 1 week after surgery to 4 weeks after surgery. At the endpoint of study, heart weight (HW)/body weight (BW) and HW/tibial length (TL) were calculated after mice were euthanized.

\subsection{Echocardiography}

Mice were anesthetized using $1.5 \%$ isoflurane, and then echocardiography was performed to assess left ventricle (LV) wall and chamber dimensions, and systolic and diastolic function using a Mylab 30CV (Esaote S. P.A, Genoa, Italy) equipped with a $10-\mathrm{MHz}$ linear array ultrasound transducer. M-mode was obtained under anesthesia while heart rate and body temperature were maintained.

\subsection{Histological examination}

The arrested hearts were fixed with 10\% formalin and then sectioned transversely. After rehydration, the sections $(5 \mu \mathrm{m})$ were stained with haematoxylin-eosin (HE) or picrosirius red (PSR). The sections were observed under light microscopy, the cross-sectional areas of the myocytes were determined by a digital analysis system (Image-Pro Plus 6.0). The sections were examined blind. In each group, more than 200 myocytes were outlined. Collagen content was determined by quantification of PSR-positive area per field of view using Image-Pro Plus 6.0. Six mice each group (6 fields every animal) were used to quantify PSR staining.

\subsection{Western blot and real-time PCR analysis}

The hearts and iced cell lysates were lysed using a RIPA buffer. The protein concentrations were determined using the BCA Protein Assay Kit. After that, the protein extracts were loaded into SDS-PAGE gels and subsequently transferred to a PVDF membrane (Millipore, IPVH00010). After incubation 
with primary antibodies and secondary antibodies, the membrane was visualized with an Odyssey Imaging System. cDNA was synthesized from the total RNA extracted from the frozen heart tissues using the Transcriptor First Strand cDNA Synthesis Kit (Roche, 04896866001). Quantitative RT-PCR analysis was performed using the LightCycler 480 SYBR Green 1 Master Mix (Roche, 04707516001). All primer details were provided in Table S1. The protein levels were normalized to GAPDH. The mRNA levels were analyzed with the $2^{-\Delta \Delta \mathrm{Ct}}$ method and normalized to GAPDH.

\subsection{Cell culture and infection with recombinant adenoviral vectors}

Replication-defective adenoviral vectors under the control of the U6 promoter were used in our study. Three rat shAMPKa2 constructs were obtained from Sigma-Genosys and three Ad-shAMPKa2 adenoviruses were generated by Vigene Bioscience. We selected the one that resulted in the greatest decrease of AMPKa2 expression for further experiments. Ad-shRNA, whose sequence was GCCTGCCGTCCAAAGTTGTAA, was the non-targeting control. The cultures of neonatal rat cardiac myocytes and cardiac fibroblasts were prepared respectively as described in the previous studies [20-21]. Cells were seeded in Dulbecco's modified Eagle medium (DMEM) (GIBCO, C11995) supplemented with $10 \%$ fetal bovine serum (GIBCO, 10099), penicillin $(100 \mathrm{U} / \mathrm{ml})$ and streptomycin $(100$ $\mathrm{mg} / \mathrm{ml}$ ) (GIBCO, 15140). Bromodeoxyuridine (0.1 $\mathrm{mM}$ ) was used to prevent fibroblast contamination in neonatal rat cardiac myocytes. Purity of the cardiac fibroblast was assessed by morphologic recognition and by positive and negative staining with antibodies against Vimentin and a-actin. The purity of cultured cardiac fibroblast in our study was greater than $98 \%$. Cardiac fibroblasts in the second and third passages were used in our studies. After $48 \mathrm{~h}$, the cells were infected with Ad-shAMPKa2 or Ad-shRNA for 4 h at a MOI of 10 . Then, these cells were thoroughly washed and synchronized using serum-free medium for 12 hours. Hypertrophy of cardiac myocytes were induced by Ang II $(1 \mu \mathrm{M}, 24 \mathrm{~h})$, and cardiac fibroblasts were also subjected to Ang II $(1 \mu \mathrm{M}, 24 \mathrm{~h})$ to detect the accumulation of collagen.

\subsection{Immunofluorescence staining}

Immunofluorescence staining was used to outline cell surface. Neonatal rat cardiomyocytes were cultured on cover slips, infected with Ad-shAMPKa2 or Ad-shRNA for $24 \mathrm{~h}$, followed by stimulation with 1 $\mu \mathrm{M}$ Ang II for $24 \mathrm{~h}$. In this study, we used a-actin to stain the cells. Cells, fixed with $4 \%$ formaldehyde, were permeabilized in $0.1 \%$ Triton X-100, and stained with anti-a-actin (1:100 dilution). Alexa Fluor 488-goat anti-mouse (Invitrogen, A11017) was the second antibody used. The cross-sectional areas were calculated blind by Image-Pro Plus 6.0. In each group, more than 100 myocytes were outlined.

\subsection{Statistical analysis}

The data in our study are expressed as mean \pm SEM. Differences among groups were compared by one-way ANOVA, followed by post hoc Tukey test. Comparison between two groups was assessed by an unpaired Student's t-test. All in vivo, in vitro and imaging studies were performed blind. $P<0.05$ was considered as statistical significance.

\section{Results}

\subsection{AA protected against cardiac hypertrophy in vivo}

As shown by Figure 1, mice subjected to $A B$ developed a remarkable hypertrophy, as indicated by increased ratios of $\mathrm{HW} / \mathrm{BW}$ and $\mathrm{HW} / \mathrm{TL}$ (Figure 1A-B). Histological examination also revealed increased cross-sectional areas of cardiomyocytes in mice with AB surgery (Figure 1C-D). Mice subjected to pressure load also developed a decline in heart function with a decrease in fractional shortening (FS), and had a significant increase in LV internal diameter at end-diastole (LVIDd) (Figure 1E). Though mice without surgery subjected to AA treatment for 7 weeks had no cardiac morphological alterations (Figure S1), mice given AA after AB surgery exhibited reduced cardiac hypertrophy (Figure 1A-E). The hypertrophic markers induced by $\mathrm{AB}$, including atrial natriuretic peptide (ANP), B-type natriuretic peptide $(\mathrm{BNP})$, and $\beta$-myosin heavy chain $(\beta-\mathrm{MHC})$, were strikingly blunted in AA-treated mice (Figure 1F). Taken together, these data suggested that AA treatment could attenuate cardiac hypertrophy induced by chronic pressure overload.

\subsection{AA attenuated cardiac fibrosis induced by $A B$ in vivo}

After pressure overload, mice displayed obvious interstitial fibrosis, as indicated by increased LV collagen volume and fibrotic markers. However, the increased collagen volume was blunted by AA treatment (Figure 2A-B). This result was corroborated by subsequent analysis of mRNA levels of transforming growth factor- $\beta$ (TGF- $\beta$ ), collagen I, collagen III, connective tissue growth factor (CTGF) and fibronectin (Figure 2C-G). 
A
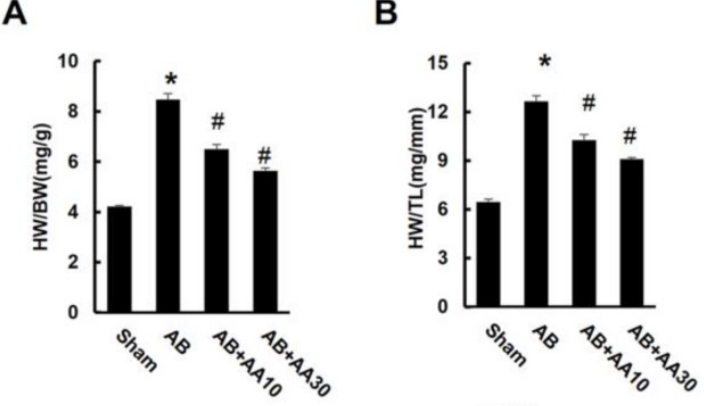

C

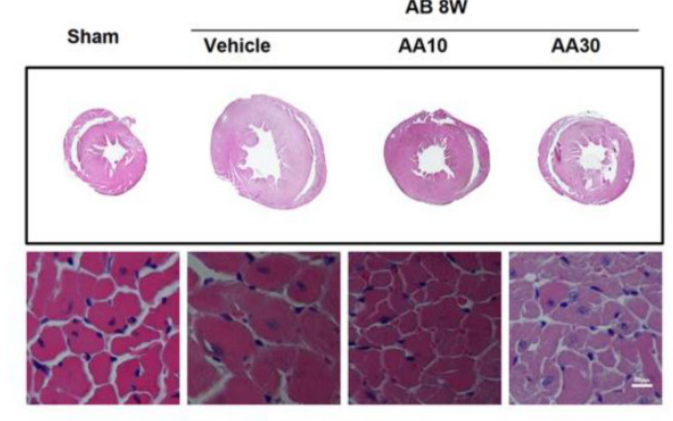

$\mathbf{F}$
D

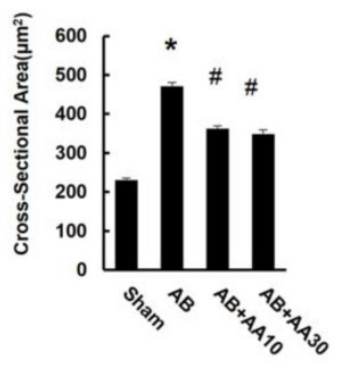

E
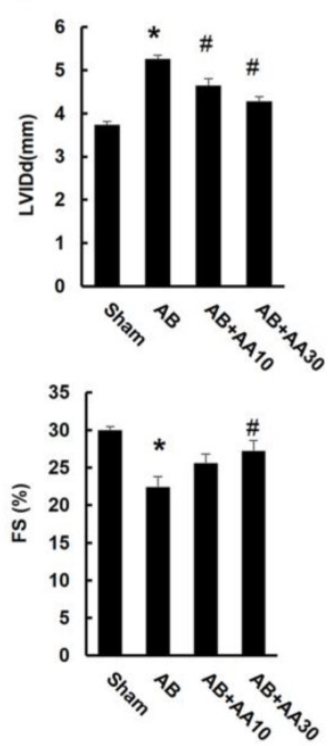

Figure 1. Asiatic acid (AA) prevented cardiac hypertrophy and dysfunction. A-B, Statistical results of heart weight $(\mathrm{HW}) /$ body weight $(\mathrm{BW}), \mathrm{HW} /$ tibial length $(T L)$ of the indicated groups $(n=15)$. C, Histological results of hypertrophic heart with or without AA. D, The cross-sectional areas of myocytes $(n=6)$. E, Echocardiographic parameters of the mice after 8 weeks of TAC with or without AA treatment $(n=10) . F$, mRNA levels of the hypertrophic markers $(n=6)$. Compared with sham, ${ }^{*} P<0.05$. Compared with $A B, \# P<0.05$.
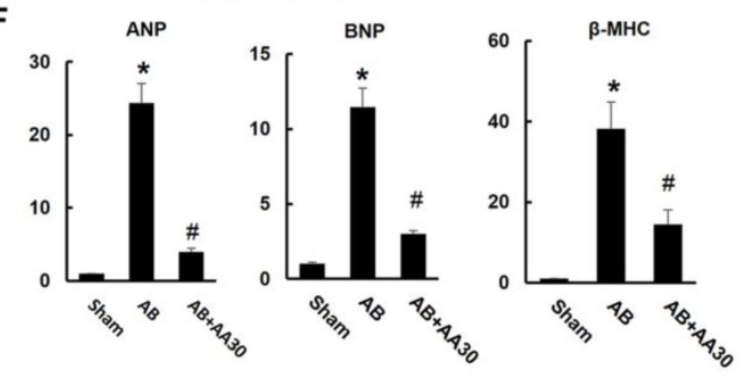

A

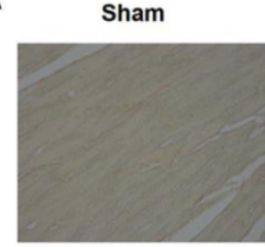

Figure 2. Asiatic acid (AA) suppressed cardiac fibrosis in vivo. A, PSR staining of hypertrophic heart with or without $A A(n=6)$. B, Collagen volume analysis of the indicated groups $(n=6)$. C-G, mRNA levels of the fibrotic markers $(n=6)$. Compared with sham, ${ }^{*} P<$ 0.05 . Compared with $A B, \# P<0.05$.
B

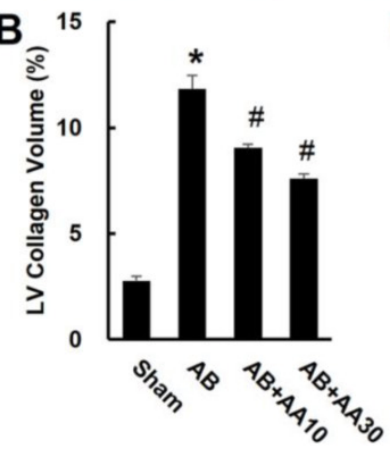

C

D
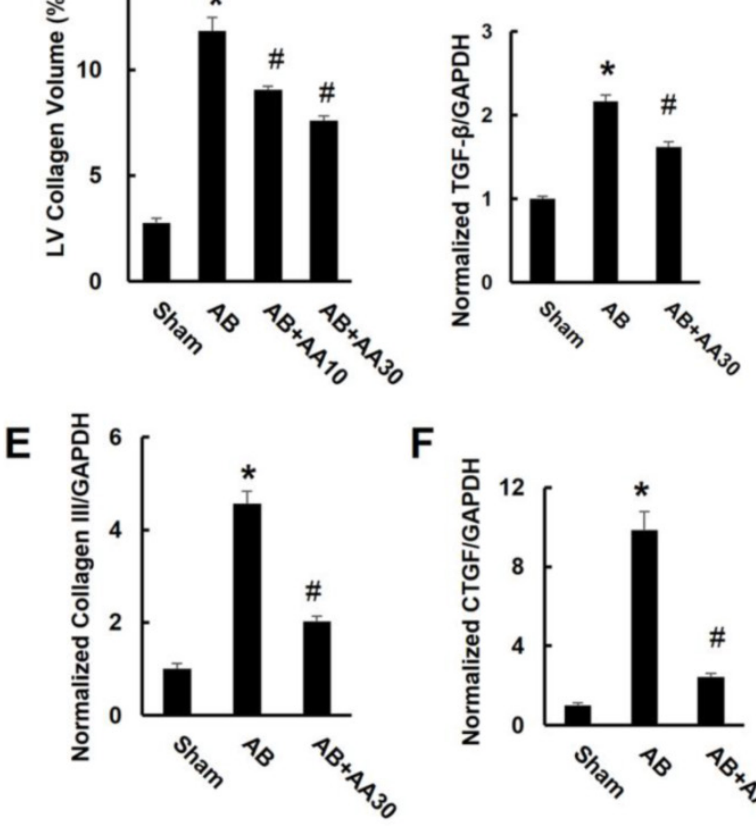

F

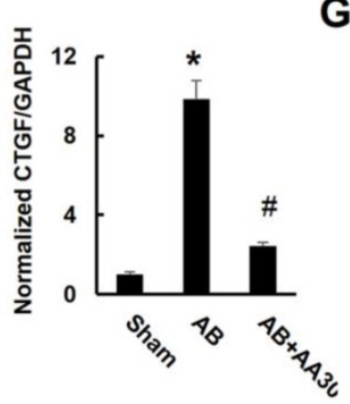

$A B$
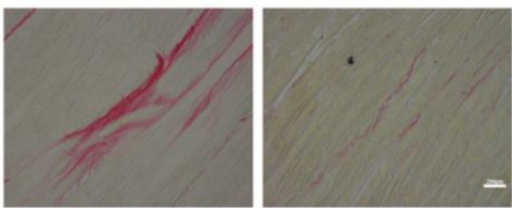

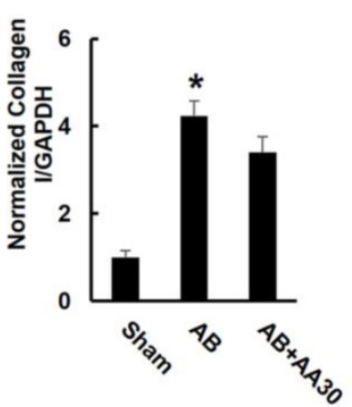

G

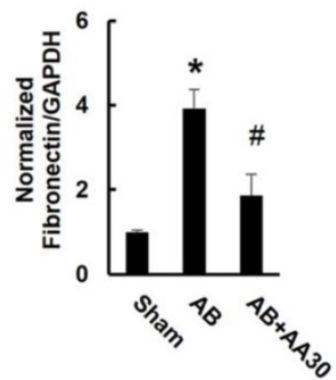


A

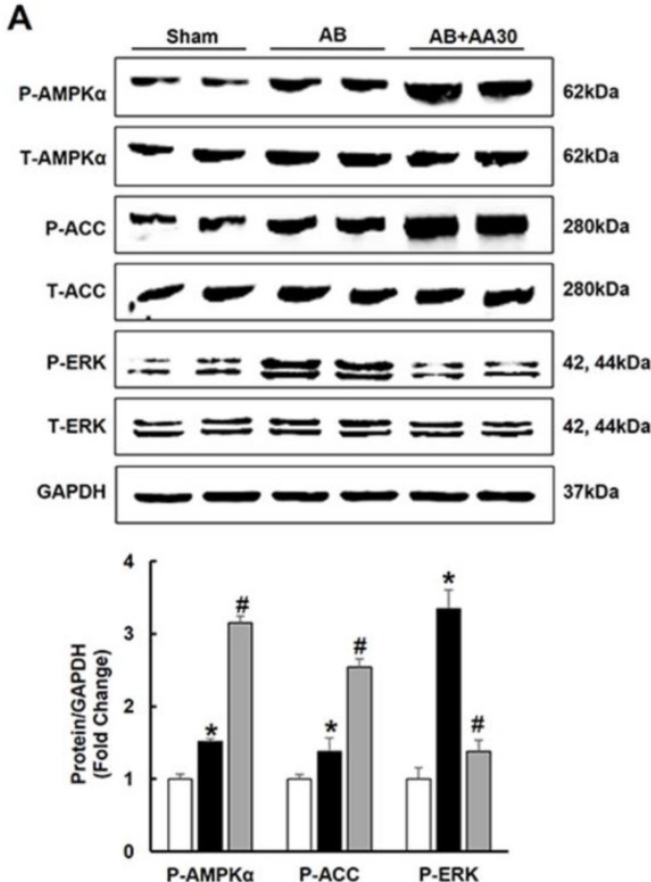

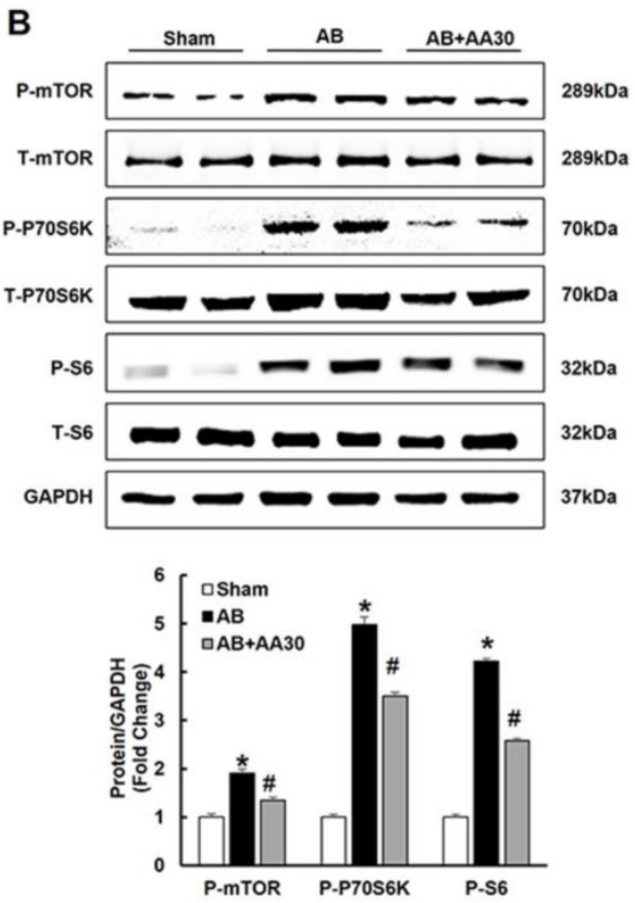

Figure 3. The effects of asiatic acid (AA) on AMPKa, mTOR and ERK signalling pathway. A-B, The protein levels of phosphorylated AMPKa and related targets in indicated groups $(n=8)$. Compared with sham, ${ }^{*} P<0.05$. Compared with $A B, \# P<0.05$.

\subsection{AA promoted the phosphorylation of AMPKa and attenuated $m$ TOR and ERK activation}

Consistent with our preliminary finding that AA could activate AMPKa at baseline (Figure S2), we also found that AA $(30 \mathrm{mg} / \mathrm{kg})$ further promoted the phosphorylation of AMPKa in the hypertrophic hearts (Figure 3A). Subsequently, the phosphorylation of ACC, which is the direct target of AMPKa and could reflect the activity of AMPKa [22], was detected. As expected, AA treatment also further promoted the phosphorylation of ACC (Figure 3A). Just as illustrated by Figure $3 \mathrm{~A}-\mathrm{B}$, compared with the sham group, AB-induced phosphorylation of mTOR, P70S6K, S6 and ERK were reduced by AA treatment.

\subsection{AA attenuated hypertrophy of myocytes in an AMPKa-dependent manner in vitro}

To decipher the effects of AA, neonatal rat cardiac myocytes were subjected to the administration of Ang II $(1 \mu \mathrm{M})$ to induce the hypertrophic response in vitro. We first examined phosphorylated AMPKa and found that AA $(20 \mu \mathrm{M})$ further activated AMPKa, which was also reflected by the phosphorylation of ACC (Figure 4A). Consistent with the results in vivo, the Ang II-mediated increases in P-mTOR, P-P70S6K, P-S6, and P-ERK were blocked in AA-treated group (Figure 4A). Moreover, AA treatment significantly inhibited cardiomyocyte hypertrophy, as indicated by decreased surface area (Figure 4C-D). The level of
ANP induced by Ang II was dose-dependently decreased after AA treatment (Figure 4B). To clearly illustrate the function of AMPKa, we knocked down AMPKa2 in cardiac myocytes. Though no significant differences in surface area and ANP were observed after knock down AMPKa2, depletion of AMPKa could completely offset the protective effects of AA in myocytes (Figure S3, Figure S4A, Figure 4C-E).

\subsection{AA inhibited the accumulation of collagen in an AMPKa-dependent manner in vitro}

Taken the fact that AA treatment activated AMPKa in the heart and cardiomyocytes into account, we detected the activation of AMPKa in cardiac fibroblasts. Expectedly, AA also activated AMPKa and suppressed mTOR and ERK pathways in cardiac fibroblasts (Figure 5A-B). Cardiac fibroblast directly transformed into myofibroblast, leading to the accumulation of collagen [23]. This prompted us to investigate the effects of $\mathrm{AA}$ on production of collagen. Treatment with AA profoundly attenuated accumulation of collagen I and collagen III, and conversely shAMPKa totally blocked the protective effects (Figure S4B, Figure 5C).

\subsection{AA had no protective effects on hypertrophy and fibrosis in mice subjected to CpC}

To further investigate whether AA exerted protective effects through AMPKa in vivo, mice were subjected to $\mathrm{CpC}$, a common AMPKa inhibitor [24, 
25]. Mice with $A B$ surgery manifested obvious hypertrophy and fibrosis. However, AA treatment did not prevent the development of cardiac hypertrophy and fibrosis in mice with AMPKa inhibition, which is reflected by morphological changes, echocardiographic examinations and molecular markers (Figure 6A-H). Collectively, these results suggested that the protective effects of AA were dependent upon the activation of AMPKa.

\subsection{AA lost its inhibitory effects on $m T O R$ and ERK in mice treated with CpC}

After $\mathrm{CpC}$ injection, AMPKa was no longer activated in mice with AA treatment (Figure 7A-B).
The phosphorylation levels of mTOR, P70S6K, S6 and ERK were not of statistical difference between $\mathrm{AB}+\mathrm{CpC}$ and $\mathrm{AB}+\mathrm{AA}+\mathrm{CpC}$ group (Figure 7B-C).

\subsection{AA exhibited no protective effects in AMPKa2 knockout mice}

In view of the fact that $\mathrm{CpC}$ had unspecific effects [26], AMPKa2 knockout mice were used. AA lost the protective effects in AMPKa2 knockout mice, as evidenced by the indistinguishable $\mathrm{HW} / \mathrm{BW}$, $\mathrm{HW} / \mathrm{TL}$, cross-sectional area and LV collagen volume.

A
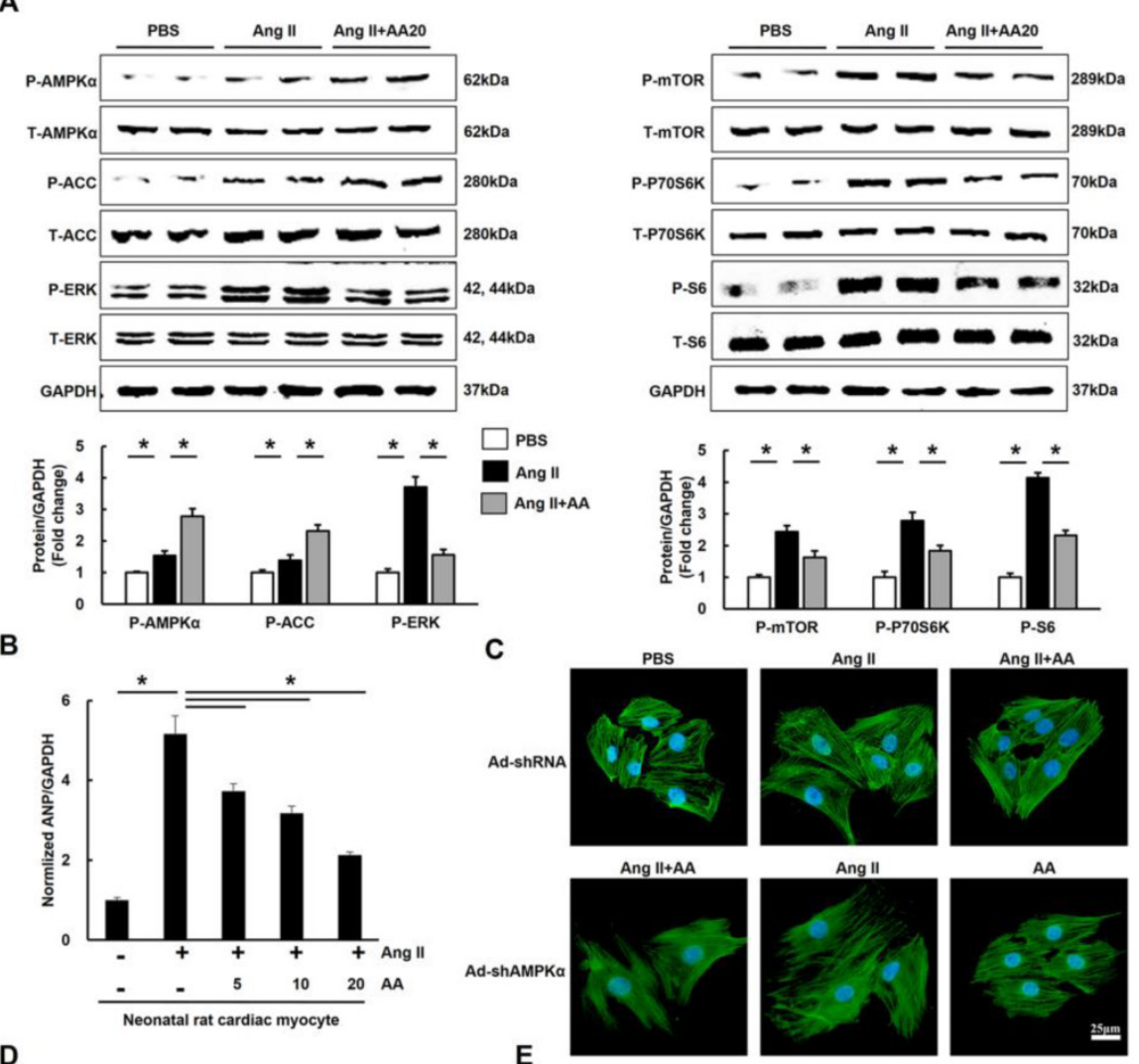

D
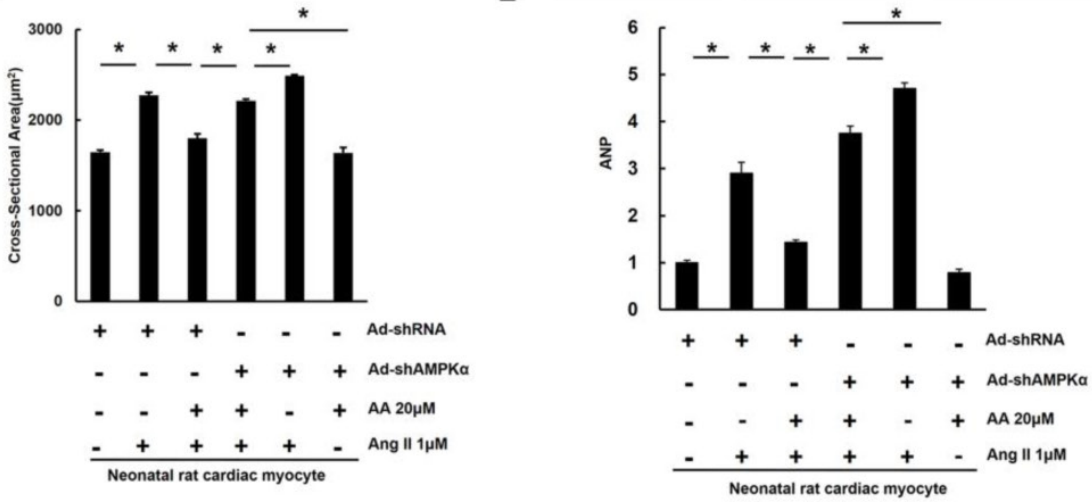

Figure 4. The effects of asiatic acid (AA) on hypertrophy of neonatal rat cardiac myocytes. A, The protein levels of phosphorylated AMPKa and related targets in cardiac myocytes $(n=8)$. B, AA inhibited increased mRNA level of ANP induced by angiotensin II (Ang II) $(n=6)$. C-D, The cross-sectional areas of myocytes $(n=6)$. E, AMPKa deficiency offset the inhibitory effect of AA on ANP $(n=6) . * P<0.05$. 

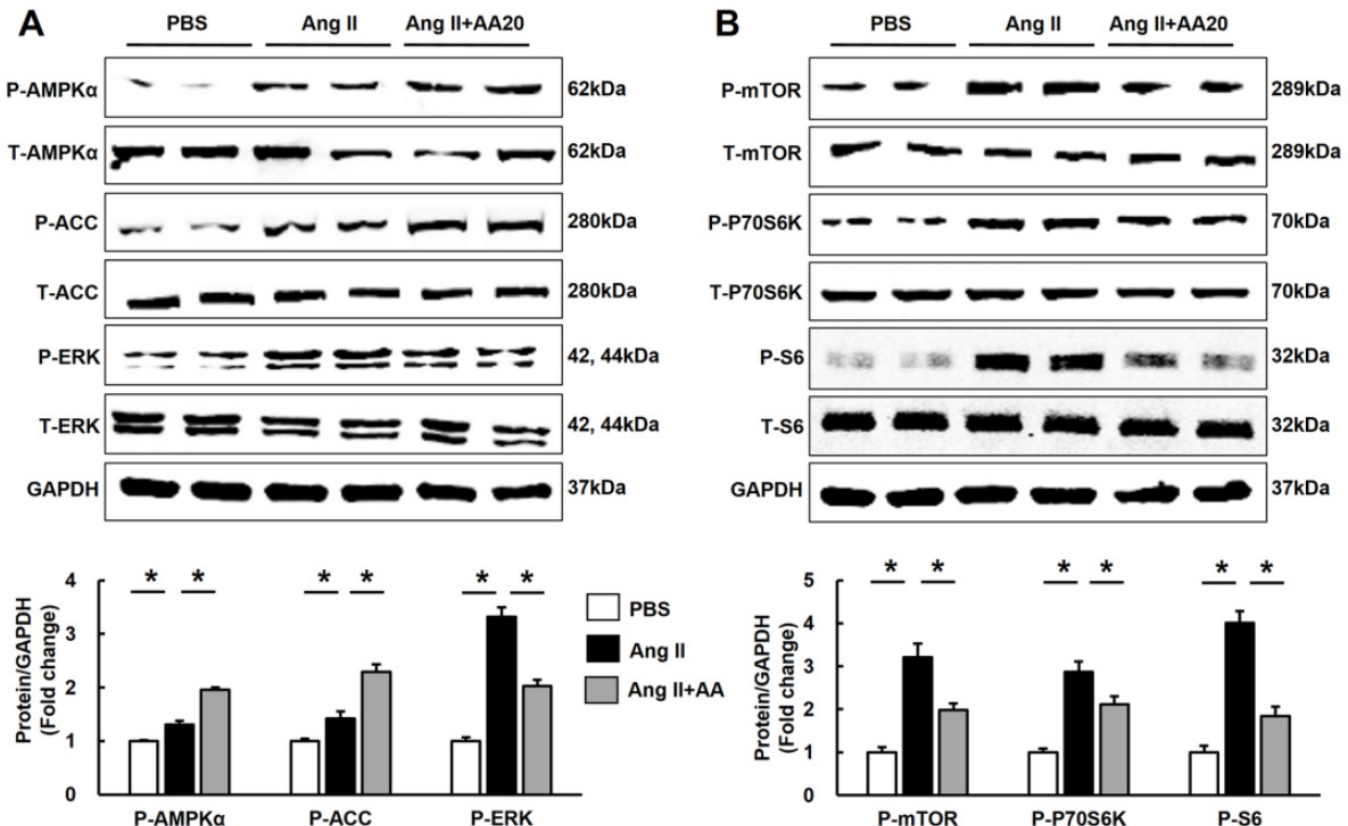

C
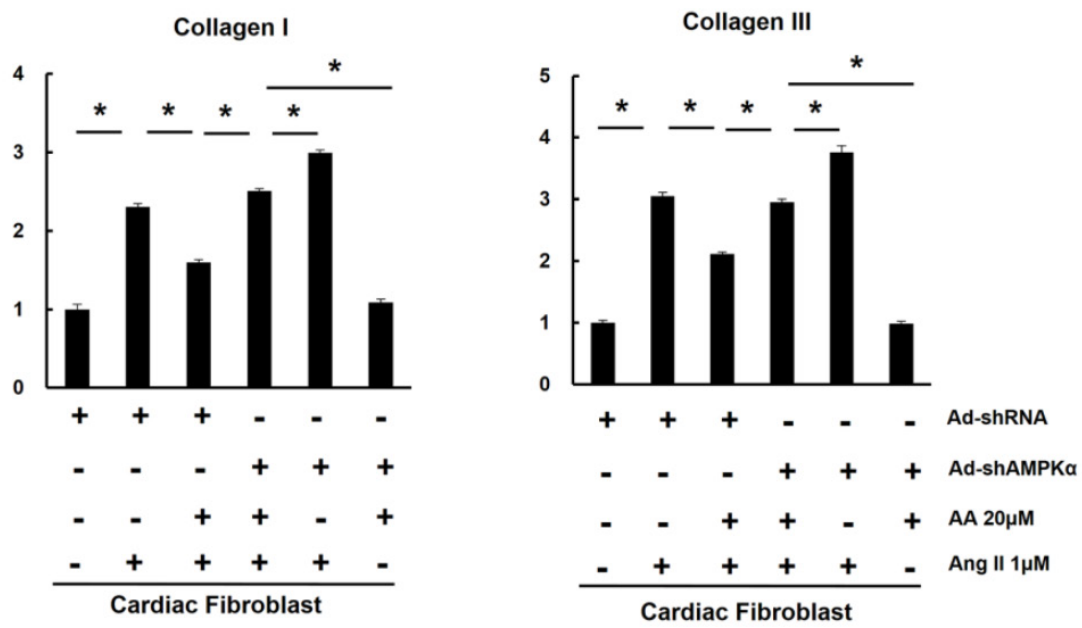

Figure 5. The effects of asiatic acid (AA) on accumulation of collagen in cardiac fibroblasts. A-B, The protein levels of phosphorylated AMPKa and related targets in cardiac myocytes $(n=8)$. C, AMPKa deficiency offset the inhibitory effect of AA on collagen I and collagen III $(n=6)$. ${ }^{*} P<0.05$.

\section{Discussion}

In the present study, we provided the evidence that AA attenuated pressure-overload induced cardiac hypertrophy in vivo and Ang II-induced hypertrophy of myocytes in vitro. AA also reduced cardiac fibrosis and accumulation of collagen. The cardio-protective effects of AA were medicated by activation of AMPKa, thus suppressing mTOR/P70S6K/S6 and ERK, which resulted in restricted hypertrophy and fibrosis, ultimately improving cardiac performance. Moreover, these protective effects of AA were abolished after AMPKa inhibition or deficiency.

The precise mechanisms by which AA exerts cardio-protective effects still remain elusive. AMPKa has been most extensively recognized as a sensor of cellular energy status, regulating process of energy metabolism during both physiological and pathological conditions [27]. Mounting reports have associated AMPKa with the hypertrophic response. It is reported that the loss of AMPKa activity is pro-hypertrophic and the activations of AMPKa by metformin and AICAR can suppress protein synthesis and hypertrophic growth in the cardiac myocyte [9, 28]. Previous report indicated that AA alleviated cardiovascular remodeling in rats with hypertension via restoration of eNOS expression, which has been proven a direct target of AMPKa [16, 29]. AA blocked TGF- $\beta$ and MAPK signalling pathways in myocytes, 
which were also regulated by AMPKa [30-32]. Therefore, the inhibitory mechanism of AA on cardiac hypertrophy was examined for its effect on AMPKa activation. Our data clearly demonstrated that AA further promoted AMPKa activation induced by hypertrophic stimuli. Using adenoviral-mediated gene transfer or the inhibitor of AMPKa, we further demonstrated that AA lost cardio-protective effects in vitro and in vivo, confirming a key role of AMPKa in the restricted cardiac hypertrophy triggered by AA.

Our study also found that AA also markedly blocked activation of mTOR in the hypertrophic hearts. Activated mTOR increases phosphorylation of P70S6K, which acts on S6, exacerbating the process of hypertrophy [33]. However, the inhibitory effects of AA on mTOR/P70S6K/S6 pathway and cardiac hypertrophy were abrogated after AMPKa depletion, which were also consistent with several previous reports. MicroRNA-451 blocked cardiac hypertrophy via activating AMPKa and inhibiting phosphorylation of mTOR [34]. Metformin, a known activator of AMPKa, reduced phosphorylation of mTOR and its downstream target P-P70S6K and protected against systolic overload-induced heart failure [35]. ERK, a key regulator activated in cardiac myocytes in response to hypertrophic stimuli [36], was also found to mediate the cardiac protection of $\mathrm{AA}$, as indicated by the fact that AA inhibited the activation of ERK and lost inhibitory effects on ERK after AMPKa inhibition or knock down. Collectively, these results indicate that AMPKa activation by AA inhibits cardiac hypertrophy partly through suppressing mTOR and ERK.
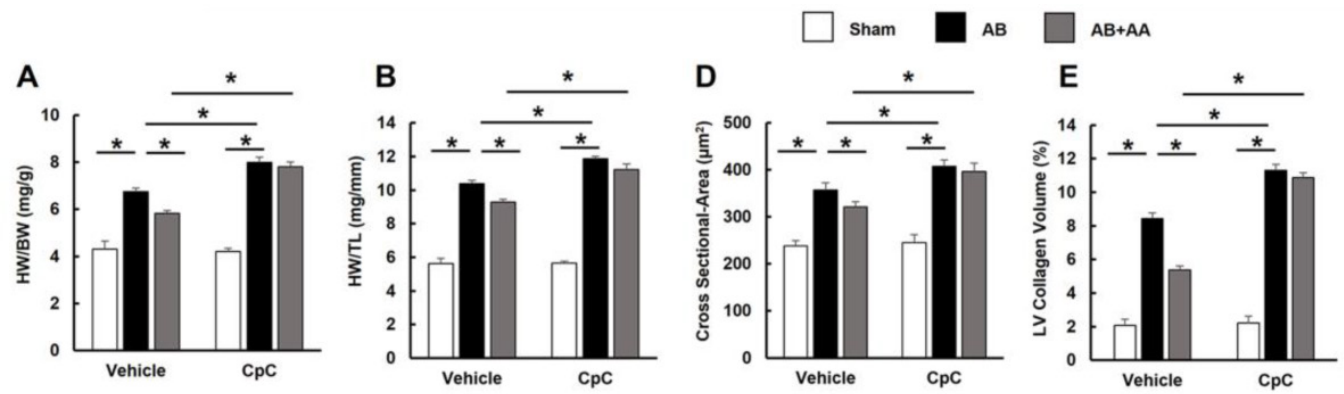

C
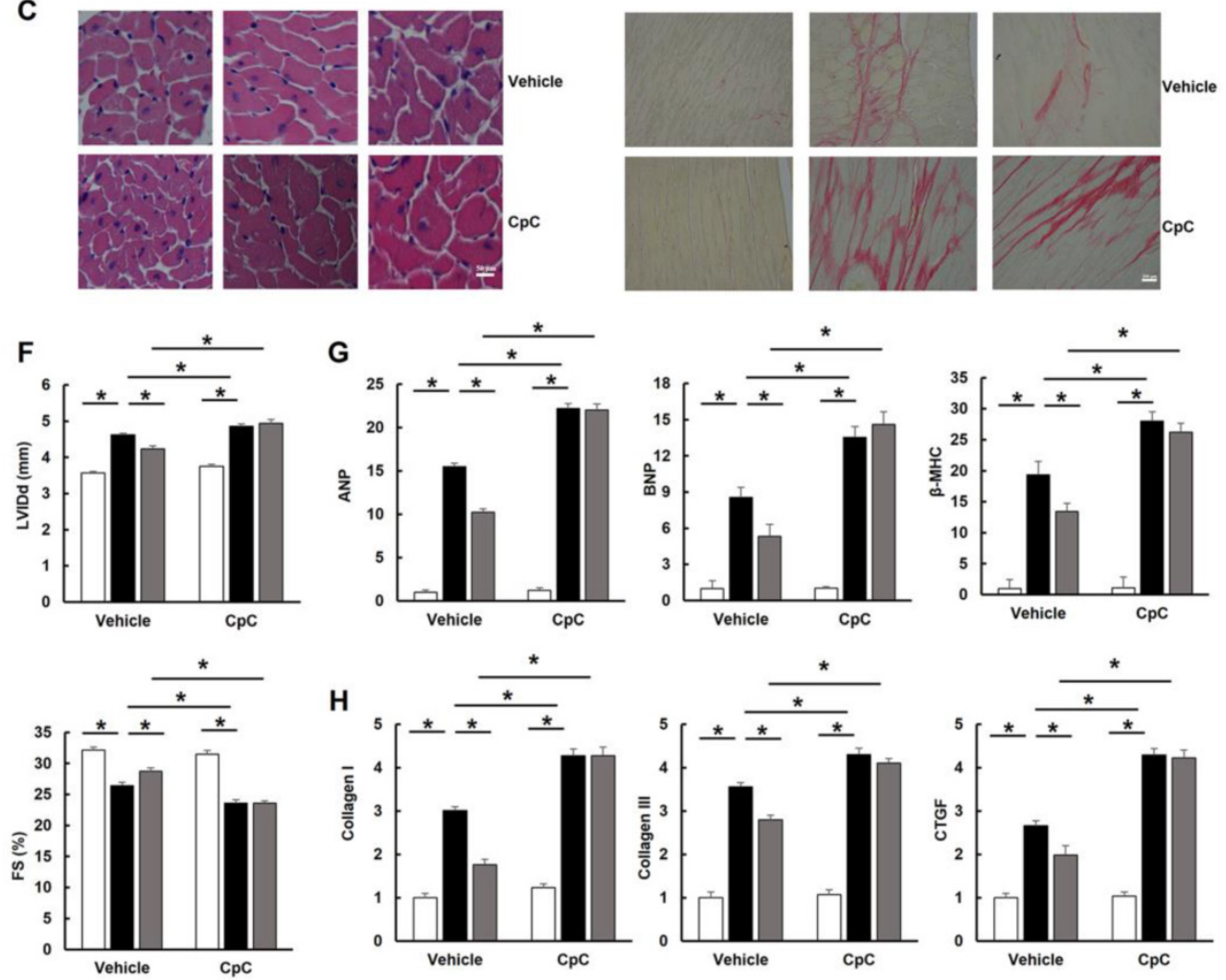

Figure 6. The effects of asiatic acid (AA) were blocked by Compound $(\mathrm{CPC})$ in vivo. A-B, Statistical results of heart weight $(\mathrm{HW}) /$ body weight (BW), HW/ tibial length $(\mathrm{TL})$ of the indicated groups $(n=12)$. C, HE staining and PSR staining $(n=6)$. D, The cross-sectional areas of myocytes $(n=6)$. E, Collagen volume $(n=6)$. F, Echocardiographic parameters $(n=8)$. G, mRNA levels of the hypertrophic markers $(n=6) . H$, mRNA levels of the fibrotic markers $(n=6) .{ }^{*} P<0.05$. 
A
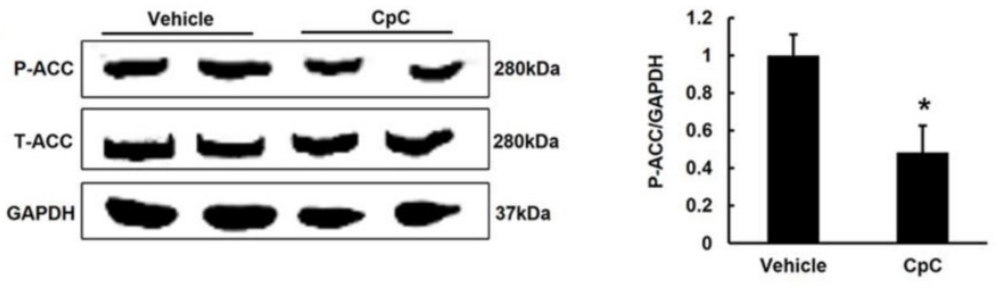

B
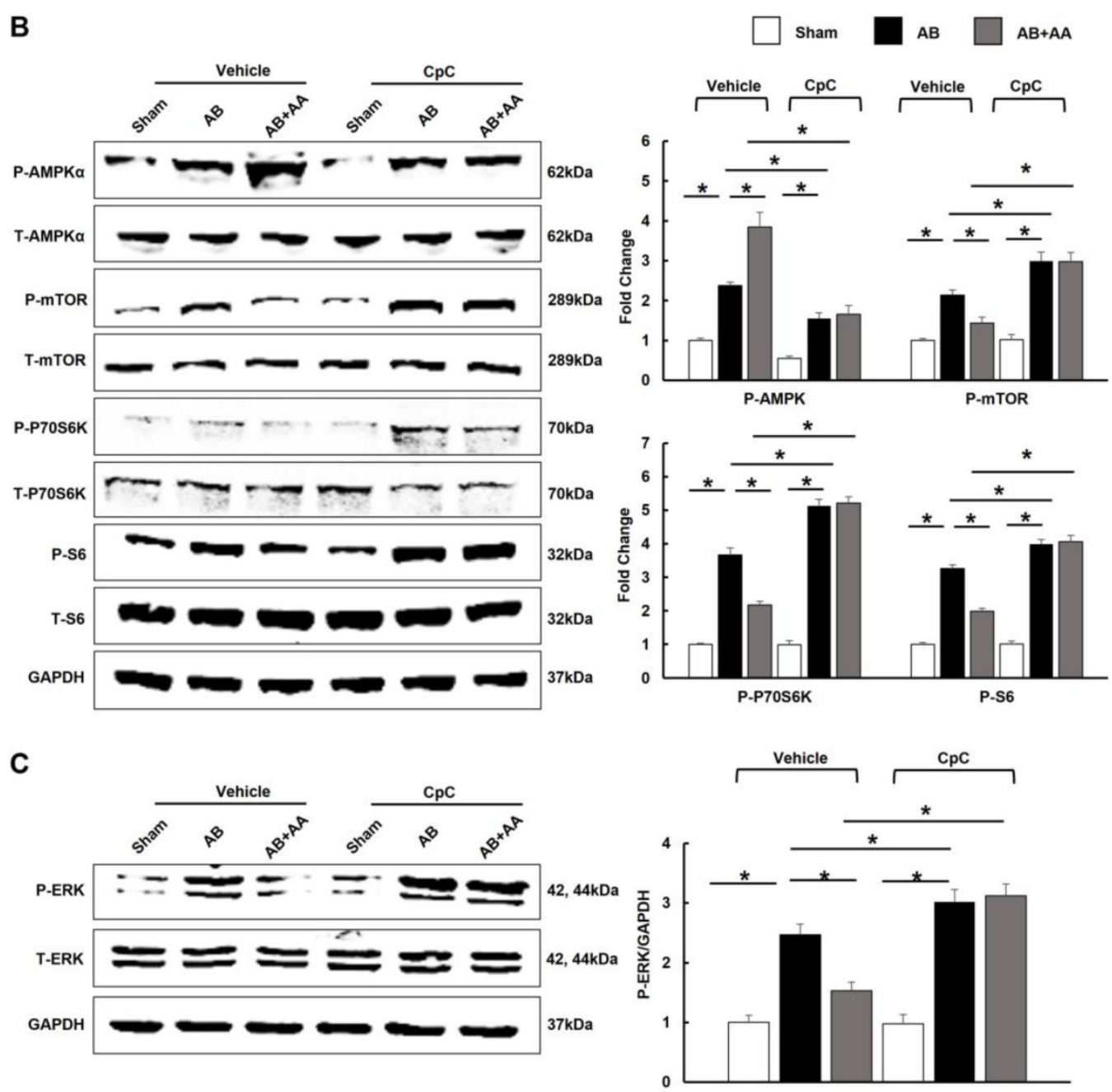

Figure 7. The effects of asiatic acid $(A A)$ and Compound $\left(C_{P} C\right)$ on AMPKa, mTOR and ERK signalling pathway. A-C, The protein levels of phosphorylated AMPKa and related targets in indicated groups $(\mathrm{n}=6)$. ${ }^{*} \mathrm{P}<0.05$.

Cardiac fibrosis, a common feature of heart diseases, is characterized by accumulation of collagen and increase of the extracellular matrix. In this study, we found that AA reduced cardiac fibrosis induced by pressure overload. Furthermore, anti-fibrotic effects of AA were evaluated in vitro using cardiac fibroblasts, and we observed that AA attenuated Ang II-induced collagen synthesis. To further elucidate the underlying mechanism, we detected effects of AA on AMPKa in fibroblast, which could suppress fibrotic actions[37], and found that AA promoted phosphorylation of AMPKa. In contrast, depletion of
AMPKa by genetic approach or a specific inhibitor disrupted the protective effects of AA in vitro and in vivo, indicating the restrained cardiac fibrosis by AA may be attributed to activation of AMPKa. mTOR/P70S6K and ERK, the downstream targets of $\mathrm{AMPKa}$, have been demonstrated to regulate the process of fibrosis[38, 39]. Limited fibrosis by AA may partly be due to the attenuation of mTOR and ERK.

In conclusion, AA protects against cardiac hypertrophy and fibrosis via AMPKa. We also show that depletion of AMPKa results in the loss of the protective effects of AA against hypertrophy and 
fibrosis. Our study provide an experimental evidence for the application of AA in the treatment of cardiac hypertrophy and heart failure. Future study aiming at elucidating the potential clinical use of AA will be of great interest.
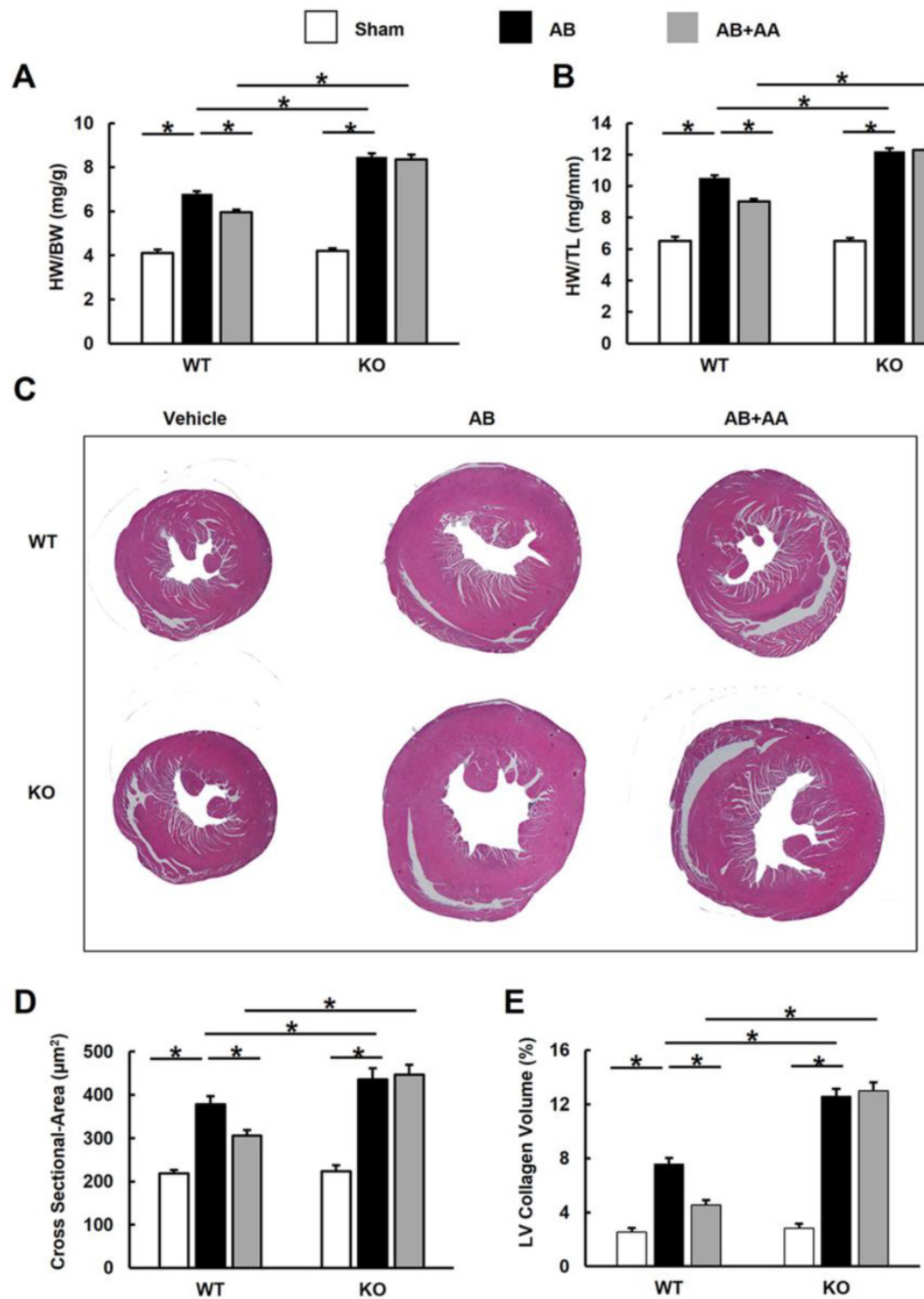

Figure 8. Asiatic acid (AA) lost the inhibitory effects on cardiac hypertrophy and fibrosis in AMPKa knockout (KO) mice. A-B, Statistical results of heart weight (HW)/ body weight (BW), HW/ tibial length $(T L)$ of the indicated groups $(n=8)$. C, Histological results of hypertrophic heart with or without AA. D, The cross-sectional areas of myocytes $(n=5)$. E, PSR staining of hypertrophic heart with or without AA $(n=5)$. WT: wild type. ${ }^{*} P<0.05$.

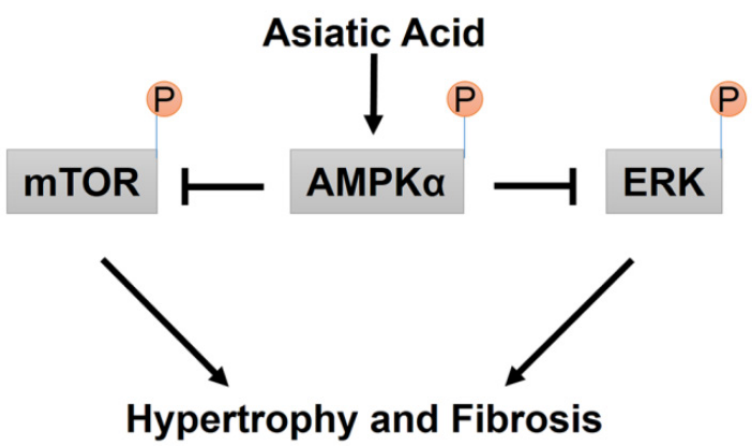

Figure 9. A proposed mechanism for the effects of AA on cardiac hypertrophy and fibrosis. 


\section{Supplementary Material}

Supplementary tables and figures. http://www.ijbs.com/v12p0861s1.pdf

\section{Acknowledgement}

This work was supported by grants from the National Natural Science Foundation of China (81270303, 81470516, 81470402), the Fundamental Research Funds for the Central Universities of China (No. 2014302020202), and the Hubei Province's Outstanding Medical Academic Leader program.

\section{Conflict of Interest}

None.

\section{References}

[1] Hill JA and Olson E N. Cardiac plasticity. N Engl J Med. 2008;358:1370-1380.

[2] Chien KR. Stress pathways and heart failure. Cell. 1999;98:555-558.

[3] de Simone G, Gottdiener JS, Chinali M and Maurer MS. Left ventricular mass predicts heart failure not related to previous myocardial infarction: The Cardiovascular Health Study. Eur Heart J. 2008; 29:741-747.

[4] McMullen JR, Sherwood MC, Tarnavski O, Zhang L, Dorfman AL, Shioi T, et al. Inhibition of mTOR signaling with rapamycin regresses established cardiac hypertrophy induced by pressure overload. Circulation. 2004;109:3050-3055.

[5] Liang F, Lu S and Gardner DG. Endothelin-dependent and-independent components of strain-activated brain natriuretic peptide gene transcription require extracellular signal regulated kinase and p38 mitogen-activated protein kinase. Hypertension. 2000;35:188-192.

[6] Ma XM and Blenis J. Molecular mechanisms of mTOR-mediated translational control. Nat Rev Mol Cell Biol. 2009;10:307-318.

[7] Meng R, Pei Z, Zhang A, Zhou Y, Cai X, Chen B, et al. AMPK activation enhances PPARalpha activity to inhibit cardiac hypertrophy via ERK1/2 MAPK signaling pathway. Arch Biochem Biophys. 2011; 511: 1-7.

[8] Ma ZG, Dai J, Zhang WB, Yuan Y, Liao HH, Zhang N, et al. Protection against cardiac hypertrophy by geniposide involves the GLP-1 receptor / AMPKa signalling pathway. Br J Pharmacol. 2016;173: 1502-1516.

[9] Chan AY, Soltys CL, Young ME, Proud CG and Dyck JR. Activation of AMP-activated protein kinase inhibits protein synthesis associated with hypertrophy in the cardiac myocyte. J Biol Chem. 2004;279:32771-32779.

[10] Yin MC, Lin MC, Mong MC and Lin CY. Bioavailability, distribution, and antioxidative effects of selected triterpenes in mice. J Agric Food Chem. 2012;60:7697-7701.

[11] Hashim P, Sidek H, Helan MH, Sabery A, Palanisamy UD and Ilham M. Triterpene composition and bioactivities of Centella asiatica. Molecules. 2011;16:1310-1322

[12] Wang X, Lu Q, Yu DS, Chen YP, Shang J, Zhang LY, et al. Asiatic acid mitigates hyperglycemia and reduces islet fibrosis in Goto-Kakizaki rat, a spontaneous type 2 diabetic animal model. Chin J Nat Med. 2015;13:529-534.

[13] Guo W, Liu W, Jin B, Geng J, Li J, Ding H, et al. Asiatic acid ameliorates dextran sulfate sodium-induced murine experimental colitis via suppressing mitochondria-mediated NLRP3 inflammasome activation. Int Immunopharmacol. 2015;24:232-238

[14] Yan SL, Yang HT, Lee YJ, Lin CC, Chang MH and Yin MC. Asiatic acid ameliorates hepatic lipid accumulation and insulin resistance in mice consuming a high-fat diet. J Agric Food Chem. 2014;62:4625-4631.

[15] Lee KY, Bae ON, Serfozo K, Hejabian S, Moussa A, Reeves M, et al. Asiatic acid attenuates infarct volume, mitochondrial dysfunction, and matrix metalloproteinase-9 induction after focal cerebral ischemia. Stroke. 2012;43:1632-1638.

[16] Bunbupha S, Prachaney P, Kukongviriyapan U, Kukongviriyapan V, Welbat $\mathrm{JU}$ and Pakdeechote P. Asiatic acid alleviates cardiovascular remodeling in rats with L-NAME-induced hypertension. Clin Exp Pharmacol Physiol. 2015;42:1189-1197.

[17] Hung YC, Yang HT and Yin MC. Asiatic acid and maslinic acid protected heart via anti-glycative and anti-coagulatory activities in diabetic mice. Food Funct. 2015;6:2967-2974.

[18] Jiang DS, Wei X, Zhang XF, Liu Y, Zhang Y, Chen K, et al. IRF8 suppresses pathological cardiac remodelling by inhibiting calcineurin signalling. Nat Commun. 2014; 5:3303

[19] Deng W, Zong J, Bian ZY, Zhou H, Yuan Y, Zhang R, et al. Indole-3-carbinol protects against pressure overload induced cardiac remodeling via activating AMPK-alpha. Mol Nutr Food Res. 2013;57:1680-1687.

[20] Sadoshima J and Izumo S. Molecular characterization of angiotensin II--induced hypertrophy of cardiac myocytes and hyperplasia of cardiac fibroblasts. Critical role of the AT1 receptor subtype. Circ Res. 1993;73:413-423.
[21] Simpson P, McGrath A and Savion S. Myocyte hypertrophy in neonatal rat heart cultures and its regulation by serum and by catecholamines. Circ Res. 1982;51:787-801.

[22] Winder WW and Hardie DG. Inactivation of acetyl-CoA carboxylase and activation of AMP-activated protein kinase in muscle during exercise. Am J Physiol. 1996;270:299-304.

[23] Leask A. Getting to the heart of the matter: new insights into cardiac fibrosis. Circ Res. 2015;116:1269-1276.

[24] Dai Y L, Huang S L and Leng Y. AICAR and Metformin Exert AMPK-dependent Effects on INS-1E Pancreatic $\beta$-cell Apoptosis via Differential Downstream Mechanisms. Int J Biol Sci. 2015; 11:1272-1280.

[25] Noyan-Ashraf MH, Shikatani EA, Schuiki I, Mukovozov I, Wu J, Li RK, et al. A glucagon-like peptide- 1 analog reverses the molecular pathology and cardiac dysfunction of a mouse model of obesity. Circulation. 2013;127:74-85.

[26] Ljubica V, Maja M, Kristina J, Vilimanovich U, Sudar E, Isenovic E, et al. Compound $\mathrm{C}$ induces protective autophagy in cancer cells through AMPK inhibition-independent blockade of Akt/mTOR pathway. Autophagy. 2011;7:40-50.

[27] Hardie DG. AMPK-sensing energy while talking to other signaling pathways. Cell Metab. 2014;20:939-952.

[28] Zhang P, Hu X, Xu X, Fassett J, Zhu G, Viollet B, et al. AMP activated protein kinase-alpha2 deficiency exacerbates pressure-overload-induced left ventricular hypertrophy and dysfunction in mice. Hypertension. 2008:52:918-924

[29] Chen Z, Peng IC, Sun W, Su MI, Hsu PH, Fu Y, et al. AMP-activated protein kinase functionally phosphorylates endothelial nitric oxide synthase Ser633. Circ Res. 2009;104:496-505.

[30] Si L, Xu J, Yi C, Xu X, Wang F, Gu W, et al. Asiatic acid attenuates cardiac hypertrophy by blocking transforming growth factor-beta1-mediated hypertrophic signaling in vitro and in vivo. Int J Mol Med. 2014;34:499-506.

[31] Chan CY, Mong MC, Liu WH, Huang CY and Yin MC. Three pentacyclic triterpenes protect $\mathrm{H} 9 \mathrm{c} 2$ cardiomyoblast cells against high-glucose-induced injury. Free Radic Res. 2014;48:402-411.

[32] Thakur S, Viswanadhapalli S, Kopp JB, Shi Q, Barnes JL, Block K, et al. Activation of AMP-activated protein kinase prevents TGF- $\beta 1$-induced epithelial-mesenchymal transition and myofibroblast activation. Am J Pathol. 2015;185(8):2168-80.

[33] Sciarretta S, Volpe $\mathrm{M}$ and Sadoshima J. Mammalian target of rapamycin signaling in cardiac physiology and disease. Circ Res. 2014;114:549-564.

[34] Kuwabara Y, Horie T, Baba O, Watanabe S, Nishiga M, Usami S, et al. MicroRNA-451 exacerbates lipotoxicity in cardiac myocytes and high-fat diet-induced cardiac hypertrophy in mice through suppression of the LKB1/AMPK pathway. Circ Res. 2015;116:279-288.

[35] Xu X, Lu Z, Fassett J, Zhang P, Hu X, Liu X, et al. Metformin protects against systolic overload-induced heart failure independent of AMP-activated protein kinase alpha2. Hypertension. 2014;63:723-728.

[36] Kehat I and Molkentin JD. Extracellular Signal-Regulated Kinase 1/2 (Erk1/2) Signaling In Cardiac Hypertrophy. Ann N Y Acad Sci. 2010;1188:96-102.

[37] Ma X, Fu Y, Xiao H, Song Y, Chen R, Shen J, et al. Cardiac Fibrosis Alleviated by Exercise Training Is AMPK-Dependent. PLoS One. 2015;10:e129971.

[38] Yu SY, Liu L, Li P and Li J. Rapamycin inhibits the mTOR/p70S6K pathway and attenuates cardiac fibrosis in adriamycin-induced dilated cardiomyopathy. Thorac Cardiovasc Surg. 2013;61:223-228.

[39] Du J, Guan T, Hui Z, Xia Y, Liu F and Zhang Y. Inhibitory crosstalk between ERK and AMPK in the growth and proliferation of cardiac fibroblasts. Biochem Biophys Res Commun. 2008;368:402-407. 